\title{
Lebensentwürfe im Spannungsfeld von Ökonomisierung, Selbstverwirklichung und unternehmerischer Nachhaltigkeit
}

\author{
Tim Thrun, Marc Casper und Maximilian Schormair
}

\author{
„Was tun Sie“, wurde Herr K. gefragt, „wenn Sie einen \\ Menschen lieben?" \\ „Ich mache einen Entwurf von ihm“, sagte Herr K., ,und \\ sorge, daß er ihm ähnlich wird.“ \\ „Wer? Der Entwurf?“ \\ „Nein“, sagte Herr K., ,,der Mensch.“ \\ Bertolt Brecht (1975, S. 33)
}

\section{Zusammenfassung}

Im Zentrum des Beitrags steht das dreiteilige Lehrkonzept des titelgebenden Seminars. In einem ersten Reflexionsblock leisten die Teilnehmerinnen und Teilnehmer überwiegend biographisch orientierte Reflexionen. Die dabei subjektiv formulierten Lebensentwürfe werden im zweiten Block durch Filmvorführungen und -diskussionen kontrastiert. Im Rahmen eines dritten Referatsblocks vertiefen die Teilnehmerinnen und Teilnehmer ihre bisherigen Überlegungen vor dem Hintergrund eines aktuell diskutierten Themas der Wirtschaftswissenschaft. Das Seminar zeichnet sich über diese drei Elemente

T. Thrun $(\bowtie)$

Helmut-Schmidt-Universität, Hamburg, Deutschland

E-Mail: thrunt@hsu-hh.de

M. Casper

Universität Hamburg, Hamburg, Deutschland

E-Mail: marc.casper@uni-hamburg.de

M. Schormair

Trinity College Dublin, Dublin, Irland

E-Mail: maximilian.schormair@tcd.ie 
hinweg durch seinen kritisch-emanzipatorischen Modus und eine ausgeprägte Lebensweltorientierung aus.

\section{Schlüsselbegriffe}

Lebenswelt · Lebensweltorientierung · Lebensentwurf · Ökonomik · Ökonomie $\cdot$ Kultur $\cdot$ Kritisch-emanzipatorische Bildung

\section{Entstehungskontext und Seminarstruktur}

„Schlagworte wie ,Work-Life-Balance“, „Nachhaltigkeit“ oder ,Achtsamkeit“ prägen derzeit den Berufsalltag. All diese Begriffe behandeln im Kern die Frage nach einem guten Leben in der Wirtschaft. Sie beschreiben einen individuellen Prozess der Suche nach einer Balance zwischen beruflichen Anforderungen einerseits und den persönlichen Werten und Lebensvorstellungen andererseits. Wir alle sind hierbei Teil einer Kultur und eines Wirtschaftssystems mit vorherrschenden Institutionen, Prozessen und Ideologien. Um ein selbstbestimmtes und zufriedenes Leben zu führen, müssen wir uns folglich sowohl mit uns selbst als auch mit der uns umgebenden ökonomischen Kultur auseinandersetzen. Meist erfolgt der Zugang zur Ökonomie jedoch in ,objektiver' Form - in Zahlen, Daten, Fakten und theoretischen Modellierungen - nicht aber aus der ganz persönlichen Perspektive individueller Lebensentwürfe [...]“ (Kursbeschreibung des Seminars „Lebensentwürfe“ im Wintersemester 2018/2019 an der Universität Hamburg).

Mit dieser auszugweise zitierten Kursbeschreibung warben wir zum Wintersemester 2018/2019 im Studienportal der Universität Hamburg um die Gunst und Anmeldung der Studierenden für das titelgebende Seminar. ,Lebensentwürfe" stand dabei als Wahlpflichtmodul des Fachbereichs Sozialökonomie Masterstudierenden ,Interdisziplinärer Public und Non Profit Studien` sowie gewerblichen Berufsschullehramtsstudierenden mit dem Zweitfach ,Betriebswirtschaftslehre` zur Auswahl.

Inhaltlich zeichnet sich ,Lebensentwürfe' also durch die Abgrenzung von einer wirtschaftswissenschaftlichen Hochschullehre aus, die die Ökonomie ausschließlich über positivistisch-objektive Wissens- und Theorieinhalte zugänglich zu machen versucht. Eine solche mathematisch-mechanistische Ökonomik (Graupe 2016, S. 341), wie sie die wirtschaftswissenschaftliche Hochschullandschaft dominiert, lässt die subjektiven Erfahrungen und Theorien der Lernenden größtenteils unberücksichtigt. Fragehorizonte zur Verknüpfung von Erfahrung und Wissen finden in ihr kaum Raum, werden nicht selten sogar als fach(ent) fremd(end) zurückgewiesen. Wir wollen ,Lebensentwürfe' als ein Konzept verstanden wissen, dass eben solchen Fragehorizonten Raum schaffen möchte. 
Als Transferprojekt kann sich ,Lebensentwürfe‘ dabei auf die mehrjährige Erfahrung aus einer verwandten Lehrveranstaltung stützen: Bereits seit 2015 wird in der Berufsschullehrerbildung der Universität Hamburg das Lehrkonzept ,Wirtschaftswissenschaft als Gegenstand Ökonomischer Bildung ‘ (,WiGÖB') umgesetzt (Casper et al. 2020). Es ist als Erfahrungsraum für eine kritisch-reflexive Auseinandersetzung mit Ökonomik aus einer spezifisch pädagogischen Perspektive konzipiert: Wie betrachten und beurteilen Bachelorstudierende des Handelslehramts ihr Hauptstudienfach Betriebswirtschaftslehre und das darin angelegte Wissen in Bezug auf ihre spätere Tätigkeit als Lehrkräfte sowie auf die im Studium intendierte pädagogische Professionalisierung? Der Modus von ,WiGÖB“ sowie einige Lernimpulse wurden mit dem Transfer in ,Lebensentwürfe' nun perspektivisch geöffnet. ,Lebensentwürfe' nimmt nicht mehr das spezifisch pädagogische Interesse angehender Lehrkräfte an der Ökonomik in den Blick, sondern verallgemeinert auf ein Erkenntnisinteresse an der Wirtschaftswissenschaft im Hinblick auf die individuelle, emanzipierte Lebensführung in einer ökonomisierten Welt.

Insofern ist ,Lebensentwürfe " über die zitierte Kursbeschreibung und die erste inhaltliche Einordnung hinaus nicht allein in kritischer Abgrenzung zu einer sogenannten ,Mainstream-Ökonomik ${ }^{\star}$ zu sehen. Vielmehr ist es uns ein Anliegen, gerade zeitgenössische und einschlägige Themen der Wirtschaftswissenschaft vor biographischen Hintergründen zum Gegenstand subjektiver Reflexionen zu machen. Die Studierenden sollen die Geltung und Bedeutung ökonomischer Theorien für die eigene Lebensgestaltung auf Basis ihrer Erfahrungen in der Ökonomie kritisch hinterfragen und sich persönlich anverwandeln, statt sich in der Aneignung positivistischer Theorie unreflektiert und oberflächlich an deren kultureller ,Reifizierung' zu beteiligen (Tafner 2018, S. 121 f.). Sie sollen Gelegenheit dazu erhalten, die vorherrschenden Rollenzuweisungen und Ideologien dieser Kultur gemessen an ihren eigenen Lebenserfahrungen und -vorstellungen differenzierter zu beurteilen und Strategien zur aktiven Mitgestaltung oder bewussten Loslösung zu entwickeln. Bildungsziel von ,Lebensentwürfe“ ist es somit, emanzipatorische Potenziale in der erfahrenen und kritisch-reflexiv betrachteten modernen Wirtschaftskultur auszuloten und alternative Konzepte ökonomischer Lebensweisen und -gewohnheiten zu entwerfen. Mit ,Lebensentwürfe“ beanspruchen wir also humanistische Bildung statt „Humankapitalbildung“ (Hirsch 2016, S. 195-197).

Methodisch umfasst das Seminar (Ablaufplan siehe in Abb.1) dazu insbesondere: 


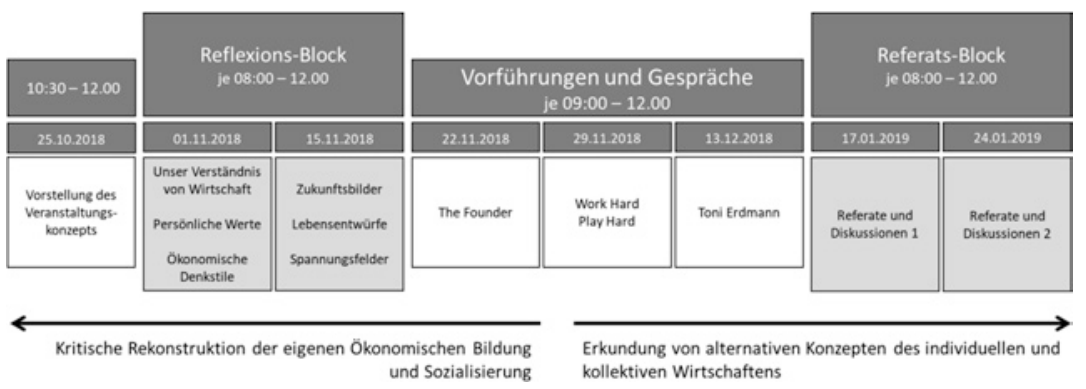

Abb. 1 Ablaufplan ,Lebensentwürfe“ im Wintersemester 2018/2019. Quelle: eigene Darstellung

- Selbstreflexion durch das Verfassen persönlicher Texte zur eigenen ökonomischen Bildung

- Kritisch-konstruktive Gruppendiskussionen zu ökonomischen Denkstilen und Strategien der wirtschaftlichen Selbstverwirklichung

- Filmvorführungen und -diskussionen, in denen die kulturelle Darstellung von Wirtschaft ergründet wird

- Vertiefung ausgewählter Themen im Rahmen von Gruppenpräsentationen

Nach einem organisatorischen Auftakt beginnt das Seminar mit zwei Blockveranstaltungen zur Reflexion der bislang erfahrenen Ökonomischen Bildung und Sozialisation. Ab der Hälfte ändert sich die Blickrichtung von einer kritischen biographischen Rekonstruktion zur Erkundung von alternativen Konzepten des Wirtschaftens. Dieser Übergang wird von Filmvorführungen und -diskussionen gestützt. Parallel dazu arbeiten die Studierenden in Kleingruppen an übergreifenden Themen, die zum Abschluss des Seminars in thesengeleiteten Präsentationen vorgestellt werden. Die Handlungsprodukte der ersten Blockphase sind Referenzpunkte für die inhaltliche Schwerpunktsetzung und Thesenentwicklung der Gruppenpräsentationen. So wird gewährleistet, dass sich die Studierenden subjektiv relevanten Aspekten und Fragen zuwenden. Das wichtigste und unkonventionellste Element dieser Veranstaltung sind daher die reflexiven Lernimpulse des ersten Blocks, die im Folgenden vorgestellt werden. 


\title{
2 Innovative Lösung im Sinne einer pluralen, sozioökonomischen Hochschulbildung
}

\subsection{Lernimpulse im Reflexionsblock}

a. Momente ökonomischer Bildung

\begin{abstract}
1.Bitte resümieren Sie und erzählen Sie von einer Erfahrung, durch die Sie , wirtschaftlich gebildet" wurden. Entscheiden Sie sich möglichst für einen ,kritischen` Moment, der Ihren Lebensentwurf beeinflusst hat. Vielleicht möchten Sie auch von einem Impuls durch einen besonderen Menschen berichten. Sie können sowohl positive als auch negative Erfahrungen schildern. Leitfragen für Ihren Text können sein:

- Was ist geschehen, wann, wo und mit welchen Beteiligten?

- Was war das ,Bildende“ an dieser Erfahrung?

- Wie hat sich Ihre Einstellung zu Wirtschaft bzw. Ihre Haltung in wirtschaftlichen Situationen dadurch verändert?

Gestalten Sie Ihren Text biographisch-erzählend (wie z. B. einen Tagebucheintrag, einen Brief an einen Freund oder eine Kurzgeschichte), kreativ und detailreich. Bitte schreiben Sie hierzu ca. eine DIN A4-Seite
\end{abstract}

Diesen Lernimpuls bereiten die Studierenden für den ersten Block vor. Dort wird unmittelbar darauf Bezug genommen:

b. Unser Verständnis von Wirtschaft

Zeit: $60 \mathrm{Min}$

1. Stellen Sie sich zu zweit gegenseitig Ihre Erfahrungen aus Lernimpuls 01 vor. Klären Sie Gemeinsamkeiten und Widersprüche. Notieren Sie gemeinsame, strittige und kritische Sichtweisen auf Wirtschaft und ökonomisches Handeln

2. Finden Sie sich zu einer Gruppe von vier Studierenden zusammen. Stellen Sie sich Ihre notierten Sichtweisen vor und visualisieren Sie ein gemeinsames Verständnis von Wirtschaft und ökonomischem Handeln auf einem Flipchartblatt

Die Studierenden tauschen sich über ihre Erfahrungen und Wirtschaftsverständnisse aus. Dieser Prozess wird dann mit einem spielerischen Impuls auf das Thema Wertvorstellungen gerichtet: 


\section{c. Persönliche Werte}

Zeit: ca. $25 \mathrm{~min}$

1.Einzelarbeit (+eine Beobachterin bzw. ein Beobachter)

a. Sortieren Sie aus den vorliegenden Karten 12 aus, die Ihnen spontan am wichtigsten erscheinen. (ca. $5 \mathrm{~min}$ )

b. Sortieren Sie aus diesen 12 Karten wiederum die 6 Karten heraus, zu denen Sie die stärksten Gefühle haben. (ca. 5 min)

c. Legen Sie sich schließlich auf die 3 für Sie wichtigsten Karten fest und bringen Sie diese in eine Rangfolge (am wichtigsten $>$ am zweitwichtigsten $>$ am drittwichtigsten). (ca. $5 \mathrm{~min}$ )

2. Vierergruppe (ca. $10 \mathrm{~min}$ )

Diskutieren Sie zu viert (je zwei Spielerinnen bzw. Spieler und zwei Beobachterinnen bzw. Beobachter)

Resümieren Sie:

- Was haben Sie gesehen, wie haben Sie sich gefühlt?

- Was ist Ihnen leichtgefallen, was schwer?

- Wie bedeutsam scheint Ihnen das Endprodukt?

Kontrastieren Sie:

- Wie passen Ihre persönlichen Werte zu Ihrem Verständnis von Wirtschaft aus Lernimpuls 02 ?

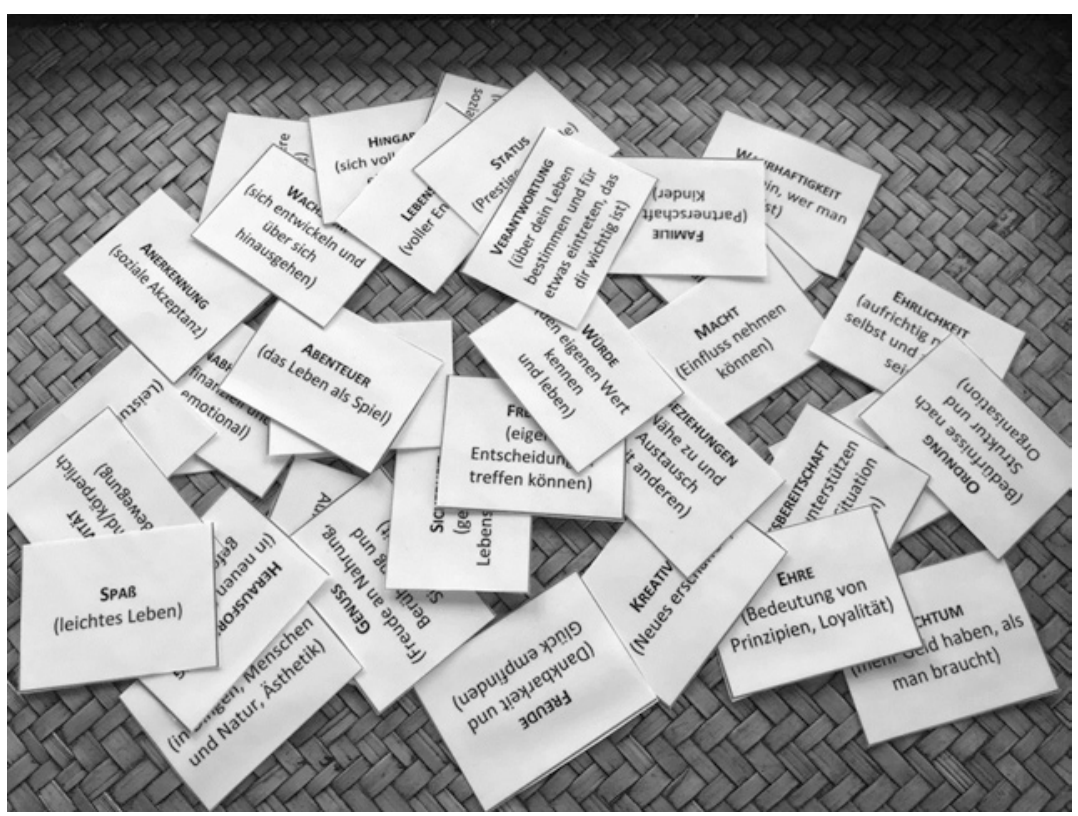

Abb. 2 Wertekarten (Auswahl, insgesamt 35). Quelle: eigene Gestaltung und Fotografie 
Das Wertespiel liefert einen Anlass, das eigene Verständnis von Wirtschaft auf persönliche Werthaltungen (siehe Abb. 2) zurückzuführen. Außerdem macht der Selektions- und Ordnungsdruck des Spiels Wertkonflikte erlebbar. Unterschiedliche Interessen und Werthaltungen werden anschließend für ökonomische Denkstile typisiert, die den Studierenden in Form archetypischer Vertreterinnen und Vertreter begegnen.

d. Archetypen ökonomischer Haltungen

Zeit: 60 Min

1. Zweiergruppen (ca. $20 \mathrm{~min}$ )

Lesen Sie die Aussagen und Zitate zu den verschiedenen Archetypen. Machen Sie sich Notizen:

a. Welchen Aussagen würden Sie zustimmen, welchen widersprechen? Welche Aussagen regen Sie zum Denken an oder erzeugen Emotionen?

b. Haben Sie Erfahrungen mit Personen gemacht, die diese oder ähnliche Aussagen verwenden (könnten)? Wie haben Sie sich zu jenen Personen verhalten?

c. Können Sie sich zu einem Archetyp oder einer Mischung aus mehreren zuordnen? Was passt zu Ihren Werten von vorhin?

d. Fallen Ihnen weitere Archetypen bestimmter ökonomischer Haltungen und typische Aussagen ein (historische oder fiktionale Figuren, Wissenschaftlerinnen und Wissenschaftler, Politikerinnen und Politiker, ,Typen', die jeder kennt)?

Diskutieren Sie Ihre Überlegungen laufend zu zweit

2. Vierergruppen (ca. $20 \mathrm{~min}$ )

Diskutieren Sie, welche Kernideen, Interessen und Werte hinter den unterschiedlichen Archetypen stehen und wie sich diese zueinander verhalten. Mit welchen Begriffen würden Sie die hier vertretenen Denkstile benennen? Visualisieren Sie Ihre Erkenntnisse auf einem Flipchart

3. Plenum (ca. $20 \mathrm{~min}$ )

Stellen Sie Ihr Flipchart im Plenum vor und beantworten Sie Rückfragen der anderen Teilnehmerinnen und Teilnehmer

Die vorgestellten ,Archetypen' sind als idealtypische Vertreterinnen und Vertreter zu verstehen, als merkmaltragende Figuren, die tradierte bzw. typische Denkstile repräsentieren (oder direkt kritisieren). Auf je einem DINA4-Blatt sind Name, Portrait und Zitate der ,Archetypen “ ausgehängt. Im Folgenden werden die jeweiligen Denkstile [in Klammern] ergänzt, die sich u. E. an diesen Beispielen thematisieren lassen:

- Milton Friedman [Neoliberalismus]

- Gordon Gecko (aus dem Film Wallstreet) [Materialismus/Egoismus]

- Johann Buddenbrook (aus Thomas Manns Roman) [klassische Kameralisten/ ehrbare Kaufleute] 
- Götz Werner [humanistische/progressive Unternehmer]

- Familie Müller-Malewski (fiktionale moderne Familie aus sozialökologischem Milieu mit einer Collage aus Popkultur-Zitaten) [Postmodernismus/Bricolage/ Eklektizismus]

- Oscar Wilde [Hedonismus]

- Arno Dübel (Trash-TV-Hartz-IV-Promi aus Hamburg) [Opportunismus, Trittbrettfahrertum]

- Dagi Bee (Youtube-Star) [Individualismus, klassischer American Dream]

- Ariadne von Schirach (mit Zitaten aus ihrem Buch ,Du sollst nicht funktionieren') [(Anti-)Konformismus]

- Karl Marx [(Anti-)Kapitalismus]

Diese Liste ist bewusst offen, um die Studierenden zur Ergänzung zu motivieren. Im Gespräch über die Archetypen soll klar werden, dass sich hinter Lebensentwürfen bestimmte Weltbilder und Denkstile befinden, für die es Begriffe und Traditionen gibt, und dass sich diese Stile oft konkurrierend gegenüberstehen.

Hiermit endet der erste Reflexionstag. Aus dessen Multiperspektivität lässt sich das Fazit ziehen, dass wirtschaftliches Handeln und ökonomische Argumentationsmuster von tief verankerten, oft latenten persönlichen Motiven und Lebensentwürfen geprägt sind. Um solche Tiefenstrukturen für den zweiten Reflexionstag sichtbar zu machen, erhalten die Studierenden den folgenden Lernimpuls:

e. Zukunftsbilder

Zeit: 2 Wochen

Im ersten Block haben wir uns mit Ihren Wirtschaftsverständnissen, persönlichen Werten und gängigen ökonomischen Denkstilen beschäftigt. Im zweiten Block wollen wir darauf schauen, wie all dies Ihre Lebensentwürfe und Ihre Selbstverwirklichung beeinflusst. In Vorbereitung darauf bitten wir Sie um einen visuellen, gern künstlerischen Beitrag: Wo sehen Sie sich in 5, 15 und 50 Jahren? Schießen Sie hierzu drei Fotos, die Ihr ,Zukunftsbild“ in je 5, 15 und 50 Jahren ausdrücken. Dabei können Sie gern kreativ und expressiv seins

Die Bilder werden am zweiten Reflexionstag mit einer Adaption der Photovoice-Methode (Wang und Burris 1997) zum Diskussionsgegenstand: 


\section{f. Zukunftsbilderflut}

Zeit: $45 \mathrm{~min}$

1. Einzelarbeit (5 min)

Wählen Sie Ihr ausdrucksstärkstes Foto, halten Sie es gut sichtbar vor sich und gehen Sie damit durch den Raum. Sehen Sie sich die Fotos aller Teilnehmerinnen und Teilnehmer an, ohne zu sprechen. Entscheiden Sie sich nach einer Weile für eine Partnerin bzw. einen Partner mit einem Foto, dass sie emotional anspricht

2. Zweiergruppen (20 $\mathrm{min})$

Tauschen Sie sich nun zu zweit über alle Ihre Fotos aus. Erklären Sie zunächst nichts zu Ihren je eigenen Fotos, sondern geben Sie ausschließlich Feedback auf die Fotos Ihres Gegenübers. Nur Rückfragen zum Verständnis sind erlaubt. Leitfragen für Ihr Feedback können sein:

- Was genau sehen Sie (nur beschreiben)?

- Welche Zustände und Ereignisse werden dargestellt (interpretieren)?

- Welche Verbindungen finden Sie zu Ihrem Leben?

- Wie bewerten Sie dieses Bild als Zukunftsvision für sich selbst (z. B. erstrebenswert, zu vermeiden, herausfordernd)?

- Was könnten/müssten Sie tun, um diese Zukunftsvision zu erreichen bzw. zu vermeiden?

3. Vierergruppen (15 min)

Finden Sie eine Vierergruppe. Berichten Sie sich gegenseitig von Ihren stärksten bisherigen Eindrücken und nominieren Sie ein Foto, das in Ihrer Gruppe am stärksten gewirkt hat

4. Einzelarbeit (5 min)

Reflektieren Sie für sich allein und machen Sie sich Notizen zu den Leitfragen:

- Was hat diese Methode insgesamt in Ihnen angeregt, was haben Sie gefühlt, gedacht, gelernt? Was ,bleibt hängen"?

- Welchen Stellenwert hatten ökonomische/wirtschaftliche Faktoren bei Ihren Überlegungen? Wie bewerten Sie das?

-Welche Verbindungen können Sie zu den Lernimpulsen des ersten Tags ziehen?

Nach dieser Auseinandersetzung mit Momentaufnahmen folgt ein systematisierender theoretischer Impuls zu den Begriffen ,Biographie“, ,Lebenslauf“ und ,Lebensentwurf‘ (unter anderem Meulemann 1999, S. 305), zur emanzipatorischen Funktion von $\{$ Auto $\}\{$ bio $\}\{$ graphie $\} \quad(=$ das (eigene $\}$ \{Leben\} \{schreiben\}; Casper 2019, S. 23) und $\mathrm{zu}$ den ökonomischen Dimensionen ,Leistung‘, ,Versorgung' und ,Eigentum‘. Vor diesem Hintergrund werden die Studierenden schließlich gebeten, ihre Lebensentwürfe als Ganzes zu visualisieren. 
g. Lebensentwürfe.

Zeit: $50 \mathrm{~min}$

1. Einzelarbeit (30 $\mathrm{min})$

Visualisieren Sie Ihren Lebensentwurf auf einem DINA4-Blatt. Gehen Sie dabei auf die ökonomischen Dimensionen Leistung, Versorgung und Eigentum ein. Ansonsten haben Sie kreative Freiheit. Im Raum finden Sie Kategorien und Leitfragen, die Ihnen beim Strukturieren helfen können, die aber nicht verpflichtend sind. Sie können auf die Ergebnisse aller bisherigen Lernimpulse zurückgreifen

2. Vierergruppen (15 min)

Finden Sie eine Vierergruppe. Stellen Sie sich gegenseitig Ihre Entwürfe vor und diskutieren Sie Gemeinsamkeiten und Unterschiede

3. Einzelarbeit ( $5 \mathrm{~min})$

Fassen Sie in einem Satz zusammen:

Welche Bedeutung hat Ökonomie in Ihrem Lebensentwurf?

Schreiben Sie Ihren Satz auf ein farbiges Post-It und hängen Sie dieses an die Wand. Versuchen Sie, zueinander passende Sätze zu gruppieren

Diese Handlungsprodukte schließen den Reflexionsblock ab und dienen den Studierenden als Referenz für die zweite Phase, die Erkundung alternativer Konzepte des Wirtschaftens. In Kleingruppen vertiefen sie selbstständig ausgewählte Themen (siehe Abschn.4). Filmvorführungen und -diskussionen ergänzen diese Phase.

\subsection{Filmvorführungen und -diskussionen}

Kennzeichnend für den Reflexionsblock war das Eintauchen in eigene und fremde Lebenswelten. Dieses Stilmittel wird über Filme fortgeführt, die den Studierenden als Projektionsfläche für Lebensentwürfe und ökonomische Denkstile dienen. Über die Identifikation mit den Protagonisten und die Deutung ihrer Handlungsmotive kann die eigene Lebensgestaltung reflektiert werden (Baacke 1978, S. 181). Die folgenden Filme wurden aufgrund ihrer ökonomischen Schwerpunktthemen und immersiven Darstellungen ausgewählt:

a. The Founder

Der Spielfilm dramatisiert die Auseinandersetzung der Gebrüder McDonald mit ihrem ersten Franchisenehmer und dem späteren ,Gründer' der McDonald's Corporation, Ray Kroc. Die Protagonisten verkörpern gegensätzliche Unter- 
nehmertypen: Dick und Mac McDonald sind Selfmade-Men, sie verkörpern einen handwerklichen, idealistischen Unternehmergeist und sind besessen vom Kleinod ihres perfektionierten „Burger-Balletts“. Ray hingegen ist statusorientiert und träumt von einem Imperium. Seine rücksichts- und gewissenlose Profitgier bedroht das ursprüngliche Geschäftsmodell der Gebrüder McDonald. An diesem Beispiel lassen sich unternehmerische Motivationen diskutieren. Den Studierenden wird hierbei die fundamentale Unterscheidung zwischen Sach- und Formalzielen als kaufmännisch-ökonomische Orientierungen bewusst (Thrun et al. 2018, S. 11).

b. Work Hard Play Hard

Diese Grimme-Preis-prämierte Dokumentation zeigt in wechselnden Episoden Arbeitsraumgestaltung, Assessment-Center, Teambuilding-Maßnahmen und Change-Management-Prozesse in Unternehmen. Der Film wirft dabei einen ungefilterten und schonungslosen Blick auf ökonomisierte Arbeitswelten. Die Studierenden werden dazu angeregt, Kulturen der Erwerbsarbeit kritisch zu kommentieren und ihre eigenen Arbeitserfahrungen zu reflektieren.

c. Toni Erdmann

In diesem Spielfilm besucht der einsame Pensionär Winfried Conradi seine vielbeschäftigte Tochter Ines in Bukarest, die dort als Unternehmensberaterin tätig ist. Bei dem Versuch, sich der ihm fremd gewordenen Tochter wieder anzunähern, taucht Winfried in die gnadenlos leistungsorientierte Businesswelt seiner Tochter ab und entwickelt, zu ihrer Karikatur, sein Alter Ego, den exzentrischen Persönlichkeitscoach Toni Erdmann. Das tragikomische Aufeinanderprallen der unterschiedlichen Lebensauffassungen von Vater und Tochter provoziert bei den Studierenden Fragen nach eigenen Zielen und Orientierungen der beruflichen und privaten Identität.

Im Anschluss an die jeweiligen Vorführungen werden die Filme im Plenum diskutiert. Nachfolgend sind beispielhaft die Diskussionsfragen für , The Founder' genannt. An ihnen wird deutlich, wie zunächst die einzelnen Handlungselemente und Charaktere besprochen und interpretiert werden, damit die Studierenden anschließend ihre eigenen Lebensentwürfe und subjektiven Vorstellungen an ihren Deutungen reflektieren können: 
The Founder - Diskussionsfragen

Ausgangssituation

Ray:

- In welcher Lebenssituation befindet sich Ray Kroc?

-Welchen Lebensentwurf hat Ray zu Beginn? Wie beschreibt das seine Frau?

- Welche Werte sind ihm wichtig?

Dick \& Mac:

- In welcher Lebenssituation befinden sich die beiden Brüder?

- Welchen Lebensentwurf haben die beiden?

- Welche Werte sind ihnen wichtig?

Das Zusammentreffen

- Was begeistert Ray an McDonalds?

- Warum erkennt er das Potenzial?

- Inwiefern war McDonalds in den 50ern das, was man heute als ,disruptive Innovation “ bezeichnen würde?

- Wie überzeugt Ray die Brüder? Warum lassen die Brüder sich auf die Zusammenarbeit ein? Welche Zweifel haben sie?

Die Konflikte

- Welchen Konflikt hat Ray mit seiner Frau? Was wünscht er sich?

- Welche Konflikte haben Ray und die Brüder?

Das Minneapolis Opening: McDonalds Nr. 1!?

- Wie verändert sich Ray durch dieses Erlebnis? Warum findet diese Veränderung statt?

Das Wachstumsproblem

- Wie unterscheidet sich Rays Wirtschaftsverständnis von dem der Brüder?

- Wie kommt das Finanzierungsproblem zu Stande und wie gehen Ray und die Brüder damit um?

The McDonalds Corporation

- Warum kommt es zum Bruch zwischen den Brüdern und Ray?

- Wieso will Ray den Vertrag verschwinden lassen? Wieso ist ihm das so wichtig?

Übergreifende Reflexionsfragen:

- Was ist für Sie die zentrale Botschaft des Films?

- Verändert sich Rays Lebensentwurf und sein Werteverständnis über den Film hinweg?

- Verändern sich die Lebensentwürfe und Werte der Brüder?

-Welche Rolle spielt Ökonomisierung in dem Film? Wie bewerten Sie das?

- Wer ist Ihrer Meinung nach der Gründer von McDonalds?

- Worin unterscheidet sich die erste von der zweiten Innovation in der Geschichte von McDonalds?

- Welchen Lebensentwurf finden Sie für sich persönlich insgesamt attraktiver?

- Was hätten Sie an Rays Stelle getan? 


\subsection{Referatsblock}

Im Anschluss an die Filmvorführungen schließt das Seminar mit dem Referatsblock. In verschiedenen Gruppen übernehmen die Studierenden jeweils eine einstündige Sequenz, die sie mit einem halbstündigen Vortrag und anschließender thesengeleiteter Diskussion füllen. Die Referatsthemen im Wintersemester 2018/2019 lauteten:

- Postwachstum und Minimalismus (unter anderem Zoellick 2017)

- Work-Life-Balance im Zeitalter der Digitalisierung (unter anderem Gregory und Milner 2009)

- Das Management der Achtsamkeit (unter anderem Arthington 2016)

- Selbstoptimierung (unter anderem Duttweiler 2016)

- Materialismus und Kommodifizierung (unter anderem Lee und Ahn 2016, Constable 2009)

- Unternehmerische Nachhaltigkeit (unter anderem Beckmann und Schaltegger 2014)

Für die Diskussionsrunden bereiten die Gruppen drei Thesen vor, die Bezüge zwischen dem jeweiligen Vortragsthema und den reflexiv-biographischen Handlungsprodukten sowie den Filmen herstellen. Die Themenbereiche besitzen eine hohe Aktualität in der postmodernen Arbeits- und Lebenswelt, wodurch sie für die ökonomisch geprägten Lebensentwürfe der Studierenden relevant sind. In der intensiven Gruppenarbeit liegen im Sinne einer kritisch-emanzipatorischen Bildung Potenziale, sich der subjektiven Beeinträchtigung und Vereinnahmung durch gesellschaftliche Anforderungen und Trends bewusst zu werden und diese kritisch zu beleuchten.

\section{$3 \quad$ Folgen, Wirkungen und Potenzial}

Die Studierenden evaluierten die Veranstaltung als methodisch und inhaltlich vielfältig, subjekt- und lebensweltorientiert und in vielerlei Hinsicht erkenntnisfördernd. Besonders positiv bewerteten sie die methodisch abwechslungsreiche Kursstruktur, sowohl im Hinblick auf die Lernatmosphäre als auch im Hinblick auf den langfristigen Lernerfolg. Sie lobten die Förderung des eigenständigen Arbeitens und bedankten sich für die Möglichkeiten, persönliche Erfahrungen mit anderen teilen sowie ihre persönlichen Werthaltungen und Einstellungen 
im Diskurs schärfen zu können. Der selbstreflexive und kritische Modus wurde von vielen als herausfordernd, aber wertvoll empfunden. Die Studierenden waren ständig dazu angehalten, ihre eigenen Denkmuster und Einstellungen zu reflektieren und diese auf ,Blindspots' hin zu untersuchen. Dieser permanente, kritische Selbstbezug im Lernprozess sei kräftezehrend, doch aufgrund des zentralen Lerneffekts, die genaue Standortbestimmung des eigenen Lebens und die Reflexion über dessen weitere Zielsetzung, auch lohnenswert. Exemplarisch dafür evaluierte eine Studierende bzw. ein Studierender ,Lebensentwürfe' augenzwinkernd als ,mindestens so viel Wert wie Therapie“.

Das besondere Potenzial entfaltet die Veranstaltung unserer Ansicht nach über ihr zentrales didaktisches Prinzip: ,Lebensentwürfe" macht die individuellen Lebenswelten und Biographien der Studierenden zum Ausgangs- und Referenzpunkt des gesamten Lernprozesses. Dabei verkommt die „Lebensweltorientierung“ nicht zu einem didaktischen „Allerweltgütesiegel“ (Oeftering et al. 2018, S. 165). Angeregt von gestaltungsorientierten Lern- und Reflexionsimpulsen, konstruieren die Studierenden in Eigenregie ihre persönlichen Lebensentwürfe. Sie werden ,zu den Autorinnen und Autoren ihrer eigenen Bildungsbiographie“ (ebd.). In diversen Diskursformen dekonstruieren sie ökonomische Imperative. Konstruktion und Dekonstruktion gehen somit Hand in Hand, individuelle Erfahrungen und ökonomische Lerngegenstände werden unmittelbar miteinander verknüpft. Durch eine solche ,lebensweltlich orientierte Didaktik“ (ebd., S. 173) können die Lernenden den Einfluss ökonomischer Ideologien auf ihr Leben kritisch-konstruktiv diskutieren (Oeftering et al. 2019, S. 275) und Alternativkonzepte handlungsorientiert beurteilen.

Die Transferfähigkeit hat sich mit der positiven Evaluation von ,Lebensentwürfe' bestätigt. Sie ermutigt dazu, weitere, ähnliche Erfahrungsräume zu schaffen - für eine kritisch-reflexive, lebensweltorientierte Auseinandersetzung mit ökonomischen Lerngegenständen.

\section{Literatur}

Arthington, P. (2016). Mindfulness. A critical perspective. Community Psychology in Global Perspective (CPGP), 2, 87-104.

Baacke, D. (1978). Der Spielfilm und die Erschließung von, Geschichten“. Einige theoretische und methodologische Probleme. Medien und Erziehung, 22, 181-196.

Beckmann, M., \& Schaltegger, S. (2014). Unternehmerische Nachhaltigkeit. In H. Heinrichs \& G. Michelsen (Hrsg.), Nachhaltigkeitswissenschaften (S. 323-368). Berlin: Springer.

Brecht, B. (1975). Geschichten vom Herrn Keuner. Frankfurt/M.: Suhrkamp. 
Casper, M. (2019). Geschichten über Wirtschaft. Studentische Erzähltexte als Zugang zu Kategorien Ökonomischer Bildung. bwp@ Berufs- und Wirtschaftspädagogik - online 35, 1-28. Online: http://www.bwpat.de/ausgabe35/casper_bwpat35.pdf [29.10.2019].

Casper, M., Tramm, T. \& Thole, C. (2020): Universitäre Lehrerbildung: Kritisch-reflexiv, multiperspektivisch, gestaltungsorientiert. Konzept und Erfahrungen aus der Veranstaltung „Wirtschaftswissenschaften als Gegenstand Ökonomischer Bildung“ an der Universität Hamburg. In C. Fridrich, R. Hedtke, W. Ötsch (Hrsg.), Grenzen überschreiten, Pluralismus wagen. Perspektiven sozioökonomischer Hochschullehre. Wiesbaden: Springer VS.

Constable, N. (2009). The Commodification of Intimacy. Marriage, Sex, and Reproductive Labor. Annual Review of Anthropology, 38, 49-64.

Duttweiler, S. (2016). Nicht neu, aber bestmöglich. Alltägliche (Selbst)Optimierung in neoliberalen Gesellschaften. Aus Politik und Zeitgeschichte, 65, 27-32.

Graupe, S. (2016). „Gefangene der Bilder in unseren Köpfen“. Die Macht abstrakten ökonomischen Denkens. Allgemeine Zeitschrift für Philosophie, 41, 341-364.

Gregory, A., \& Milner, S. (2009). Editorial: Work-life Balance. A Matter of Choice? Gender, Work and Organization, 16, 1-13.

Hirsch, M. (2016). Die Überwindung der Arbeitsgesellschaft. Eine politische Philosophie der Arbeit. Wiesbaden: Springer VS.

Lee, M. S. W., \& Ahn, C. S. Y. (2016). Anti-Consumption, Materialism, and Consumer Well-Being. The Journal of Consumer Affairs, 50, 18-47.

Meulemann, H. (1999). Stichwort: Lebenslauf, Biographie und Bildung. Zeitschrift für Erziehungswissenschaft, 2, 305-324.

Oeftering, T., Oppermann, J. \& Fischer, A. (2018). Gestaltbarkeit aller Lebensbereiche. Der Bildungswert der Lebensweltorientierung für die sozioökonomische Bildung. In T. Engartner, C. Fridrich, S. Graupe, R. Hedtke \& G. Tafner (Hrsg.), Sozioökonomische Bildung und Wissenschaft. Entwicklungslinien und Perspektiven (S. 163-184). Wiesbaden: Springer.

Oeftering, T., Oppermann, J., Fischer, A., \& Hantke, H. (2019). Lebensweltsituationsorientierte Konstruktion von Lernaufgaben in der sozioökonomischen Bildung. In C. Fridrich, R. Hedtke, \& G. Tafner (Hrsg.), Historizität und Sozialität in der sozioökonomischen Bildung (S. 265-291). Wiesbaden: Springer VS.

Tafner, G. (2018). Ökonomische Bildung ist Sozioökonomische Bildung. Grundlagen der Didaktik einer Reflexiven Wirtschaftspädagogik. In T. Engartner, C. Fridrich, S. Graupe, R. Hedtke \& G. Tafner (Hrsg.), Sozioökonomische Bildung und Wissenschaft. Entwicklungslinien und Perspektiven (S. 109-140). Wiesbaden: Springer.

Thrun, T., Casper, M. \& Bauer, N. (2018). Ökonomisches Handeln oder Kaufmännisches Handeln? Eine Inhaltsanalyse studentischer Essays über Kriterien des ,Wirtschaftens'. bwp@ Berufs- und Wirtschaftspädagogik - online 35, 1-23. http://www.bwpat.de/ausgabe35/thrun_etal_bwpat35.pdf. Zugegriffen: 29. Okt. 2019].

Wang, C., \& Burris, M. A. (1997). Photovoice: concept, methodology, and use for participatory needs assessment. Health education \& behavior: the official publication of the Society for Public Health Education, 24, 369-387.

Zoellick, J. (2017). Postwachstum. Unser Leben nach dem Wachstum. München: isw Institut für sozial-ökologische Wirtschaftsforschung e. V. 
Open Access Dieses Kapitel wird unter der Creative Commons Namensnennung 4.0 International Lizenz (http://creativecommons.org/licenses/by/4.0/deed.de) veröffentlicht, welche die Nutzung, Vervielfältigung, Bearbeitung, Verbreitung und Wiedergabe in jeglichem Medium und Format erlaubt, sofern Sie den/die ursprünglichen Autor(en) und die Quelle ordnungsgemäß nennen, einen Link zur Creative Commons Lizenz beifügen und angeben, ob Änderungen vorgenommen wurden.

Die in diesem Kapitel enthaltenen Bilder und sonstiges Drittmaterial unterliegen ebenfalls der genannten Creative Commons Lizenz, sofern sich aus der Abbildungslegende nichts anderes ergibt. Sofern das betreffende Material nicht unter der genannten Creative Commons Lizenz steht und die betreffende Handlung nicht nach gesetzlichen Vorschriften erlaubt ist, ist für die oben aufgeführten Weiterverwendungen des Materials die Einwilligung des jeweiligen Rechteinhabers einzuholen.

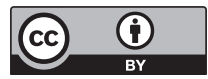

